\title{
LIGHT HARNESSING BY ALGAE: FROM FUNDAMENTAL INVESTIGATIONS TO LIGHT-BASED BIOTECHNOLOGIES
}

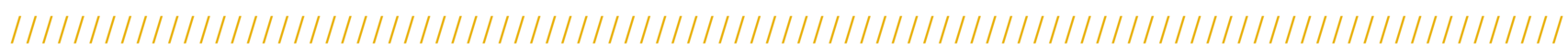

\section{Damien SORIGUE}

Aix-Marseille University, CEA, CNRS, Institute of Biosciences and Biotechnologies, BIAM Cadarache, 13108 Saint-Paul-lez-Durance, France *Damien.sorigue@cea.fr

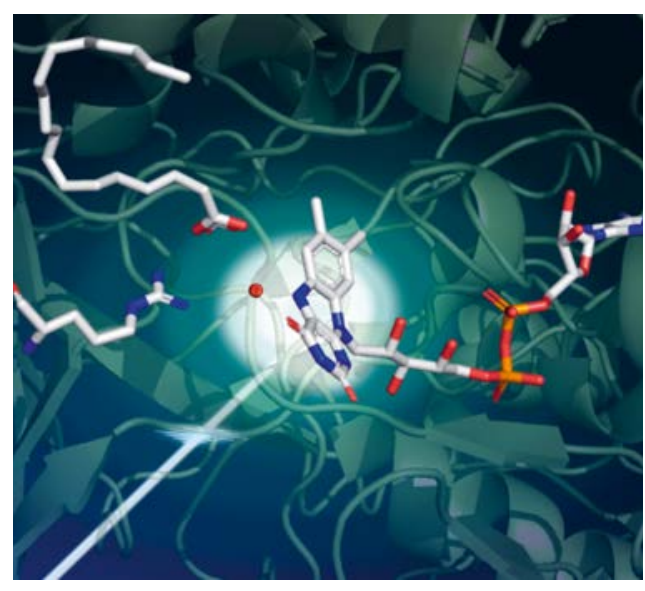

Light is the most abundant source of energy on earth and is used by photosynthetic organisms to drive the synthesis of organic molecules. Light also allows the catalysis of few enzymes, the photoenzymes. Among them, the fatty acid photodecarboxylase (FAP) isolated from microalgae converts fatty acids into hydrocarbons. We present here our understanding of the role of hydrocarbons produced by FAP in vivo, the catalytic mechanism of the FAP and its potential biotechnological applications.

https://doi.org/10.1051/photon/202111044

This is an Open Access article distributed under the terms of the Creative Commons Attribution License (http://creativecommons.org/licenses/by/4.0), which permits unrestricted use, distribution, and reproduction in any medium, provided the original work is properly cited.

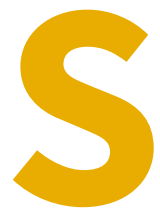

unlight powers all life processes, warming our planet and providing energy for photosynthetic life. In photosynthetic organisms, light is harvested by the photosynthetic chain that allows the conversion of photon energy into chemical energy, which can then be used by the Calvin cycle and cellular metabolism to fix $\mathrm{CO}_{2}$ and generate the organic compounds essential for growth. Among the photosynthetic organisms, we find plants, algae, cyanobacteria, and microalgae. It is not surprising that we find in these organisms, biological systems allowing the perception and the use of the light. However, known enzymes capable of directly using photon energy for their catalysis are still very rare. With the exception of photosystems, to date only three natural photoenzymes have been identified, DNA photolyase involved in DNA repair, light dependent protochlorophylide oxidoreductase, and FAP that convert fatty acids to hydrocarbons [1] (figure 1). All these photoenzymes are found in microalgae but FAP is the only photoenzyme of obvious biotechnological interest. In this perspective, we will address our current understanding of the role of hydrocarbons produced by FAP in microalgal cell, the catalytic mechanism of the FAP and its potential biotechnological applications.

\section{FATTY ACID \\ PHOTODECARBOXYLASE IS A PHOTOENZYME SPECIFIC TO ALGAE}

We have previously shown that various species of microalgae naturally produce hydrocarbons and that this production is strictly dependent on the presence of light [2]. The hydrocarbon-forming enzyme present in 
the microalgae Chlorella variabilis has been identified by partial purification of the activity and a proteomic analysis. The enzyme allows the direct conversion of fatty acids into hydrocarbons plus $\mathrm{CO}_{2}$. This enzyme contains a light-absorbing FAD cofactor and we have shown that excitation of FAD by light is required for each catalytic cycle. This photoenzyme was named fatty acid photodecarboxylase (FAP). FAP belongs to a family of enzymes that is present in a wide array or organisms, from animals to bacteria. However, the FAP subgroup seems only present in algae and most algal genomes known to date possess a gene encoding a FAP protein [3].

The biological function of the hydrocarbons produced by FAP in microalgae is still not clear but a function linked to photosynthetic membranes is likely. Indeed, in the model microalgae Chlamydomonas reinhardtii, it has been shown that

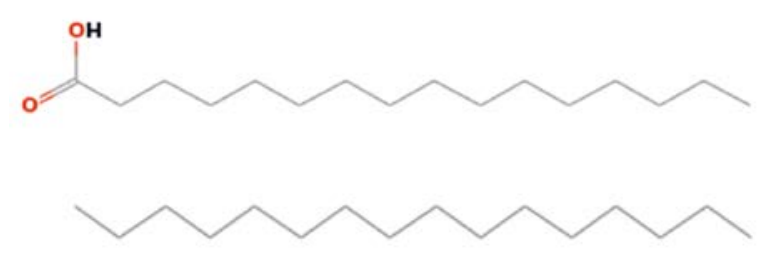

\section{Fatty acid \\ (Hexadecanoic acid)}

\section{Hydrocarbon \\ (hexadecane)}

Figure 1 : Chemical structures of fatty acid and a hydrocarbon.

all fatty-acid derived hydrocarbons are produced by FAP and $>90 \%$ of them are localized to the membrane fraction of the chloroplast, the subcellular organelle that harbors the photosynthetic membranes [3]. Besides, in a Chlamydomonas reinhardtii mutant in which the gene encoding FAP has been knocked out, the photosynthetic activity of the algae has been found to be reduced under specific conditions (namely after cold acclimation when light intensity varies). Moreover, a phylogenetic analysis in algae indicates that the gene encoding FAP was mostly conserved during evolution of algal lineages but was always absent when the photosynthetic capacity was lost. Hypotheses about the role of hydrocarbons produced by FAP in photosynthetic membranes include the regulation of the overall fluidity of the membranes in response to specific conditions of light and temperature, a role in the association/dissociation between specific proteins of the photosynthetic apparatus or the action as a chemical signal in membranes.

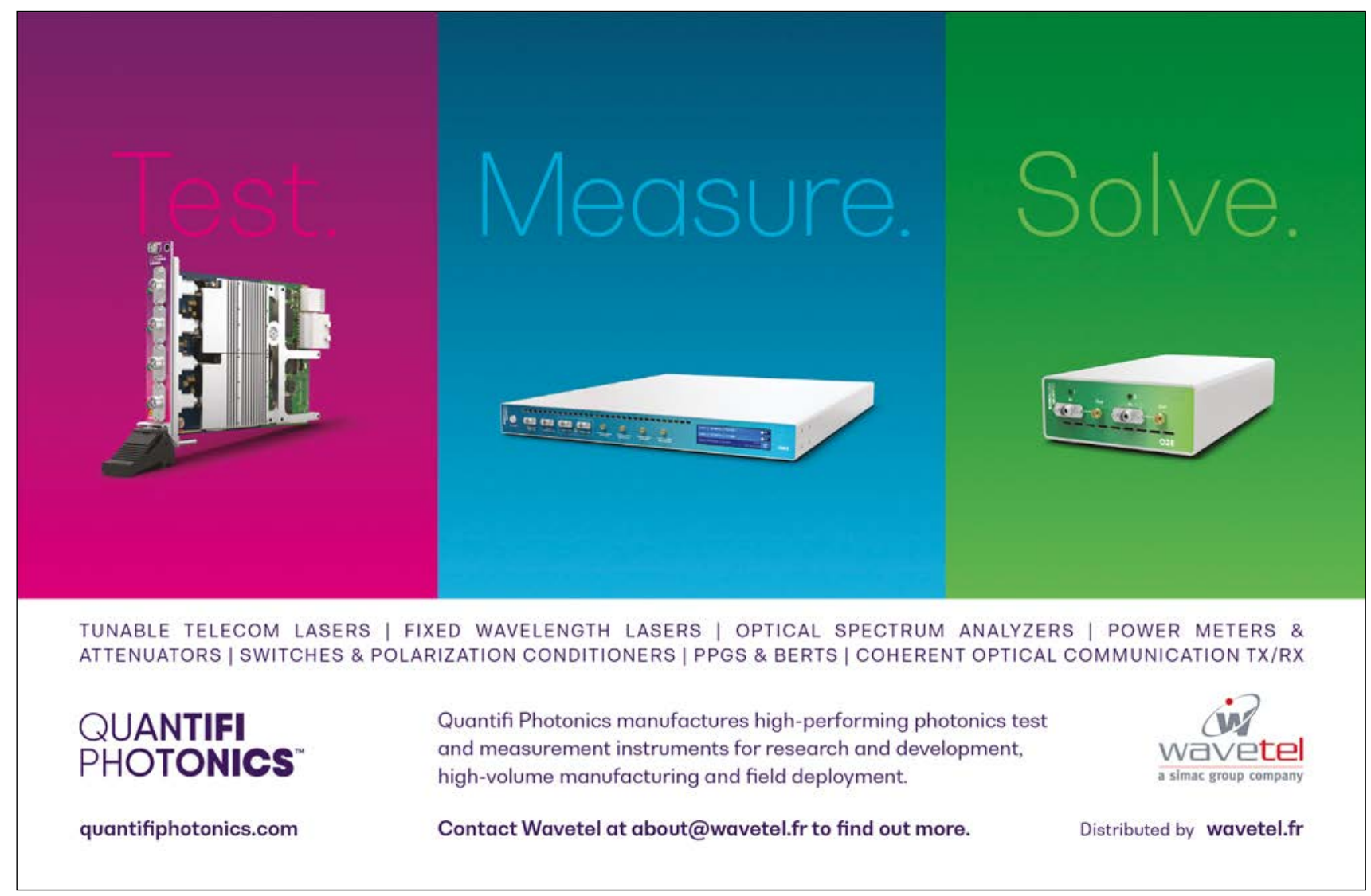




\section{MECHANISM OF}

\section{A HYDROCARBON-PRODUCING}

\section{PHOTOENZYME}

Photoenzymes offer the possibility to study ultrafast processes taking place in living organisms, by allowing a synchronization of the whole sample by light. We can therefore study reactions such as proton transfer, electron transfer, bond breakage [4]. A recent study has improved our knowledge of the enzymatic mechanism of FAP by studying the wild type enzyme as well as mutants by numerous biophysical approaches such as static spectroscopy, time-resolved spectroscopy, and cryotrapping approaches associated with spectroscopy, as well as static and time-resolved crystallography. Combined with quantum mechanics approaches, the researchers proposed the mechanism illustrated and detailed in figure 2 [5].

This work has provided important results regarding the catalytic mechanism of FAP but other questions arise. FAP has a certain substrate specificity. And to date, we don't know if this substrate specificity is related to the binding in the active site or to a difference in quantum yield. Other questions arise concerning $\mathrm{CO}_{2}$ and its conversion to bicarbonate, the researchers were surprised by the conversion of $\mathrm{CO}_{2}$ to bicarbonate within the enzyme by a catalytic process [5], we still do not know how the bicarbonate will leave the protein.

Finally, it has been shown that the lifetime of FAD RS is associated with a proton transfer in the protein, identifying the amino acids involved in this proton relay would also be an advance in the understanding of the mechanism.
We hope that in the near future, these questions can be answered by different biophysical approaches.

\section{POTENTIAL \\ BIOTECHNOLOGICAL APPLICATIONS OF FAP}

Hydrocarbons are at the heart of our economy. Produced from the distillation of petroleum, they are used in most sectors, from chemicals to cosmetics and transport. This exhaustible resource is unequally

Figure 2: Upon light excitation (1), forward Electron Transfer occurs in 300 ps from the fatty acid anion to 1 FAD* (observed by ultrafast fluorescence and transient absorption spectroscopies) and leads to its quasi-instantaneous decarboxylation (2), as observed by Time Resolved Infrared spectroscopy and Time Resolved Serial Femtosecond crystallography and supported by the computed absence of an energy barrier. Back Electron Transfer occurs in 100 ns from FAD•- (presumably to the alkyl radical) and results in formation of red-shifted (re-) oxidized flavin FADRS; the Hydrogen/Deuterium Kinetic Isotope Effect suggests that back Electron Transfer is coupled to and/or limited by a Proton Transfer. Cryotrapping Fourrier Transform Infrared spectroscopy experiments suggest arginine as the final proton donor to the alkyl (3). Concomitantly, most $\mathrm{CO}_{2}(\sim 75 \%)$ is transformed (4) to bicarbonate as indicated by Time Resolved Infrared spectroscopy and cryotrapping Fourrier Transform Infrared spectroscopy. FADRS disappears in 3ms (5) with a Hydrogen/Deuterium Kinetic Isotope Effect $>3$, indicating coupling to Proton Transfer. Upon alkane release (6), new substrate binds (7). About $25 \%$ of the formed $\mathrm{CO}_{2}$ is not transformed to bicarbonate, likely because it migrates away from the active site within $100 \mathrm{~ns}$, leaving the protein in $1.5 \mathrm{~ms}\left(4^{\prime}\right)$. In this minor fraction, arginine (R451) should reprotonate at latest in the $\sim 3 \mathrm{~ms}$ step (5). Changes after individual steps are marked in red; time constants are for RT.
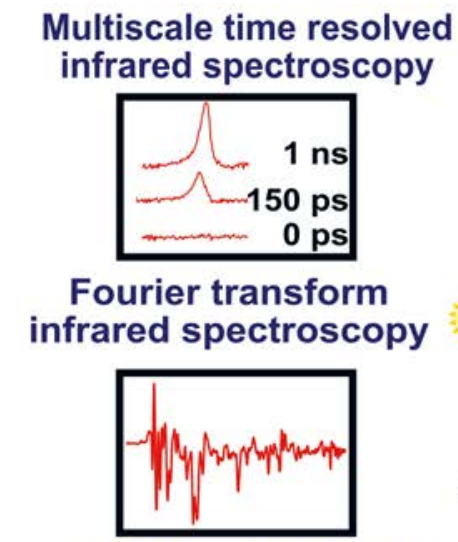

Time-resolved serial femtosecond crystallography

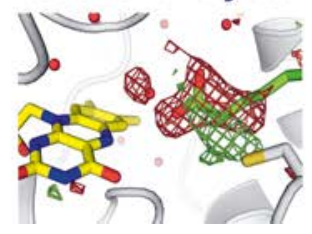

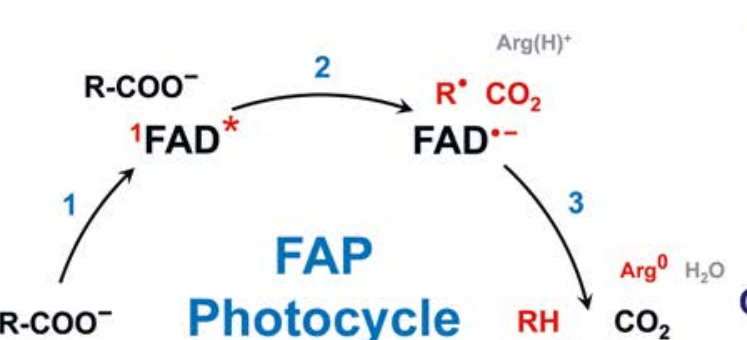

R-COO- Photocycle $\mathrm{RH}^{-} \mathrm{CO}_{2}$

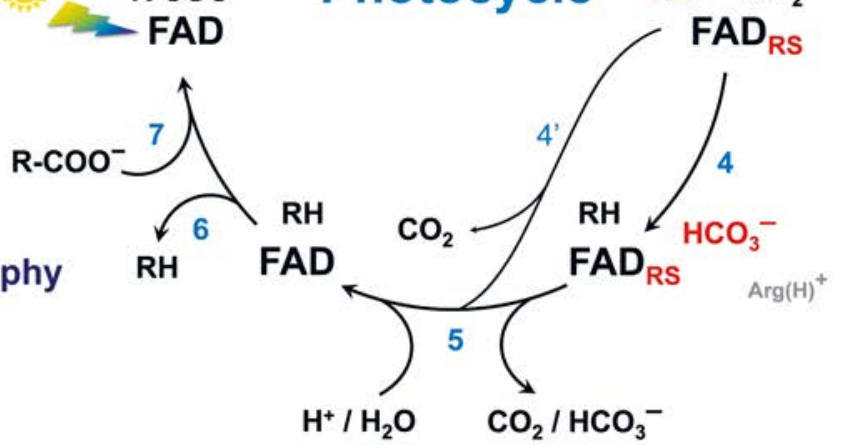

\section{Transient absorption spectroscopy}

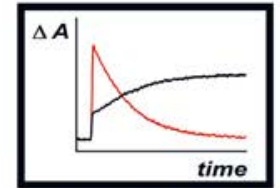

Quantum chemistry

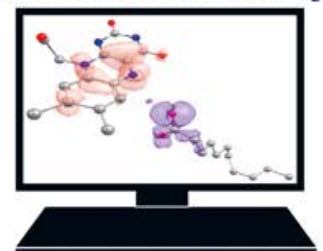

X-ray crystallography

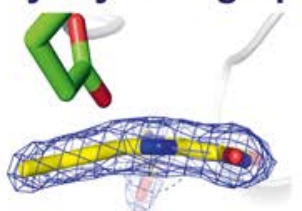


distributed on the surface of the globe and variations in oil prices have a strong impact on the economy. Chemically, hydrocarbons are made solely of carbon and hydrogen. Alkanes are saturated hydrocarbons, alkenes are unsaturated hydrocarbons. The combustion of these hydrocarbons in thermal engines produces $\mathrm{CO}_{2}$ and is associated with global warming by amplifying the greenhouse effect. It is interesting to note that global warming is not only related to the emission of $\mathrm{CO}_{2}$ during combustion but rather to the natural resource used, renewable or not. Fossil petroleum was formed from the accumulation of organic matter (mainly plants and phytoplankton, i.e. microalgae and cyanobacteria) that was buried underground and due to pressure and temperature conditions was transformed into petroleum. Formed between 20 million and 350 million years before our era, the carbon contained in petroleum remained trapped until the $19^{\text {th }}$ century. Since then, this carbon has been massively released into the atmosphere by industry and transports.

An alternative to fossil fuels could be the production of hydrocarbons directly by photosynthetic organisms as hydrocarbons can directly replace the fossil compounds. Numerous studies on FAP have shown its ability to act on fatty acids substrates from 4 to 20 carbons allowing the production of hydrocarbons ranging from propane to nonadecane. Moreover, enzymatic cascades have been realized and by combining FAP with a lipase, it is then possible to directly convert lipids (triolein) into fatty acids (octadecenoic acid) and then fatty acids into hydrocarbons (heptadecene). With a two-step cascade using homogeneously dissolved triolein and lipase, production of heptadecenes was observed in presence of FAP [6]. Finally, using structure-based engineering researchers identified regions in the natural substrate binding channel of FAP. With targeted mutagenesis they generate enzymes variants with an increased activity on short chain fatty acids. These enzymatic mutants that produce propane and butane from butanoic and pentanoic acids respectively was finally express in a photosynthetic microorganism thus allowing a direct conversion of $\mathrm{CO}_{2}$ into Hydrocarbons [7].

\section{CONCLUSION}

In conclusion, FAP is an excellent enzymatic model for biotechnology research. Although the best hydrocarbon productions have been observed in non-photosynthetic organisms, we hope that the use of FAP in photosynthetic organisms and in particular in oleaginous algae will be able to develop and allow a more direct conversion of $\mathrm{CO}_{2}$ into hydrocarbon. For the fundamental research on enzymatic catalysis, the understanding of the mechanistic details will bring new knowledge of enzymatic reactions. We imagine that it will facilitate the development of more efficient enzymatic variants allowing to bring alternative solutions to the use of fossil compounds.

\section{REFERENCES}

[1] D. Sorigué et al., Science 357, 903-907 (2017)

[2] D. Sorigué et al., Plant Physiol. 171, 2393-2405 (2016)

[3] S. Moulin et al., Plant Physiol. 186, 1455-1472 (2021)

[4] D. Zhong, Curr. Opin. Chem. Biol. 11, 174-181 (2007)

[5] D. Sorigué et al., Science 372, eabd5687 (2021)

[6] Huijbers et al., Angew. Chem. Int. Ed. Engl 57, 13648-13651 (2018)

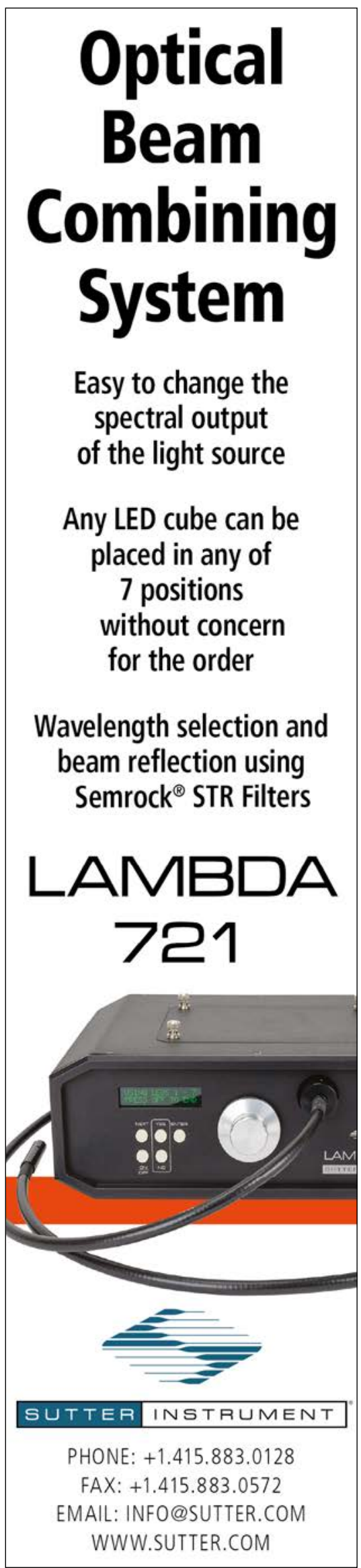

